\title{
RESEARCH AND COMMENTARY
}

\section{Factor Structure of the PASS Cognitive Tasks: A Reexamination of Naglieri et al. (1991)}

\author{
John H. Kranzler \\ University of Florida \\ Li-Jen Weng \\ National Taiwan University
}

\begin{abstract}
This study further investigated the factor structure of a battery of tasks hypothesized to measure the constructs of the planning, attention, and simultaneous-successive (PASS) processes model of human cognition. Confirmatory factor analysis was used to compare the fit provided by the PASS model with two alternative models of mental ability not examined by Naglieri, Das, Stevens, and Ledbetter (Journal of School Psychology, 29, 1-17, 1991): (1) a PASS $+g$ hierarchical model, and (2) a revised (PA)SS model, with separate Simultaneous and Successive factors and a combined Planning/ Attention factor. Contrary to the results of Naglieri et al. (1991), this study did not find that the PASS model provides a significantly better fit to the data than either of the alternative models. In fact, the results of this study suggest that the original PASS model provides an improper factor solution for these data. Not only were individual differences in the Planning and Attention factors found to be indistinguishable, but a substantial $g$ among the PASS tests was discovered. According to the goodness-of-fit indices examined in this study, the best fit was provided by the revised (PA)SS model. In sum, the results of this study suggest that further refinement of the PASS theory or tests, or perhaps both, is necessary.
\end{abstract}

Keywords: Cognitive processes; Intelligence; Intelligence measures; Psychometric $g$.

The planning, attention, and simultaneous-successive (PASS) processes theory of human cognition has recently received increased attention as an innovative model for assessing individual differences in intelligence (e.g., Das, 1992; Das \& Varnhagen, 1986; Naglieri, 1989; Naglieri \& Das, 1988; Naglieri, Das, \& Jarman, 1990; Naglieri, Prewett, \& Bardos, 1989). Derived from Luria's $(1966,1973,1980)$ work in neuropsychology, the PASS model proposes that three functional units of the brain are integrally related to information processing: (1) attention, which entails the distribution of cognitive resources and effort; (2) information coding, which comprises the use of

Received November 18, 1992; final revision received March 15, 1993.

Address correspondence and reprint requests to John Kranzler, PhD, School Psychology Program, University of Florida, 1403 Norman Hall, Gainesville, FL 32611-2053. 
simultaneous and successive processes to acquire, store, and retrieve information; and ( 3 ) planning, which involves the formulation, selection, and regulation of mental plans. The functional units are held to be "dynamic and interactive," yet "physiologically and functionally distinct" (Naglieri et al., 1990, p. 429).

According to Naglieri (1989), conventional intelligence (IQ) tests such as the Wechsler Intelligence Scales for Children-Third Edition (WISC-III) (Wechsler, 1991) and the Kaufman Assessment Battery for Children (K-ABC) (Kaufman \& Kaufman, 1983) are based on outmoded and narrow conceptions of intelligence. These tests are also seen to adequately measure only the simultaneous and successive processes related to information coding. Das (1992) states that omitting measurement of the planning and attention functional units results in (a) the ranking of individuals on a "unidimensional scale of merit" (p. 138) and (b) ineffectual differential diagnosis and remediation of cognitive deficits. Therefore, to improve upon the state of the art in intellectual assessment, Das and Naglieri are developing the Cognitive Assessment System (CAS), a new test of intelligence based on a battery of cognitive tasks hypothesized to measure each of the PASS constructs (e.g., Naglieri et al., 1989; Naglieri \& Das, 1988, 1990).

Perhaps the most important piece of evidence that has been presented in support of the validity of the PASS tests comes from a recent study by Naglieri, Das, Stevens, and Ledbetter (1991). In this study confirmatory factor analysis was used to compare the relative fit provided by the PASS model with several "traditional" models of mental ability, namely:

(1) $g$, or general intelligence, proposed by Spearman and recently reworked by Jensen (1986); (2) memory versus reasoning, best exemplified in Jensen's distinction between Level I and Level II abilities; (3) verbal versus nonverbal, as distinguished by Wechsler's intelligence tests; and (4) verbal-spatialspeed, a variant of verbal versus nonverbal with the addition of speed. (p. 4)

The PASS model was found to provide a significantly better description of the tests' factor structure than the other models. Naglieri et al. (1991) concluded that the "PASS model of cognition, based on broad functional divisions of the brain, is a viable alternative to previous conceptualizations of intelligence" (p. 14).

However, in confirmatory factor analysis when competitive alternative models are not considered, the results can be used to support virtually any model. In Naglieri et al. (1991), one could argue that at least two of the alternative models of intelligence are not very traditional. First of all, Jensen has never maintained that individual differences in mental ability are best represented by $g$ alone (A. R. Jensen, personal communication, September 30,1992 ), as suggested by Naglieri et al. (1991). Spearman even acknowledged the existence of group factors in addition to $g$ (see Spearman, 1927). 
Indeed, as Jensen (1987a) clearly states, "the $g$ factor is but one of many, although it is an especially important one" (p. 196). A similar case can be made for the memory-versus-reasoning model, or Jensen's (1974) Level I/ Level II theory. Jensen (1987b) maintains that "the purpose of Level I/Level II was never to function as a theory of the organization of the whole broad range of human mental ability" (p. 359), but to explain one aspect of group differences in test performance. He also states that Level I and II should not be regarded as "factors of ability in the strict factor analytic sense, but as two categories of tasks" (Jensen, 1987b, p. 357). Level I and Level II represent the endpoints of a continuum on which mental tasks can be arranged according to the information processing complexity required for successful task completion (Vernon, 1981). Hence, the relatively poor fit to the data provided by the $g$ and memory-versus-reasoning models in Naglieri et al. (1991) is neither unexpected nor convincing evidence of the superiority of the PASS model.

In addition to comparing the PASS model with these nontraditional models of intelligence, Naglieri et al. (1991) overlooked what is perhaps the most competitive alternative model of the structure of mental ability. According to Jensen (1986), "there is now a high degree of consensus among researchers studying abilities that a hierarchical factor analysis provides the best representation of the correlational structure of human abilities" (p. 307). If so, then the relative fit provided by a hierarchical model (cf. Gustafsson, 1984; Carroll, 1993; Undheim \& Gustafsson, 1987) must be examined before a definitive statement regarding the viability of the PASS model can be made.

The purpose of this study is to use confirmatory factor analysis to compare the fit provided by the original PASS model with two additional alternative models of mental ability, using data presented by Naglieri et al. (1991). The first alternative model is hierarchical. It is simply an extension of the PASS model to include a second-order $g$ factor (PASS $+g$ ). This model therefore has four first-order factors, corresponding to the four PASS processes, and one higher-order factor. The second alternative model is indicated by the data of Naglieri et al. (1991). Although they state that the results of their study fit "the pattern of relationships predicted by the PASS model very closely" ( Naglieri et al., 1991, p. 11), no mention is made of the fact that the correlation between the Planning and Attention factors for the Grades 5-12 group is 1.00. Given the apparent indistinguishability of these two factors with this grade group, the second alternative model is a revised PASS model, with separate Simultaneous and Successive factors and one combined Planning/Attention factor, which we called (PA)SS. Note that in confirmatory factor analysis a submodel with three first-order factors and one second-order factor is simply "identified." In other words, a hierarchical model with three first-order factors and one second-order factor will have the same degrees of freedom and chisquares that a nonhierarchical model with three intercorrelated first-order factors would have. This precludes a statistical comparison of the relative fit to the data provided by a (PA)SS $+g$ hierarchical model. 
The second-order psychometric $g$ of the (PA)SS model, however, can be examined by applying the Schmid-Leiman orthogonalization procedure (Schmid \& Leiman, 1957) to the first-order correlation matrix of the (PA)SS model. The Schmid-Leiman procedure, the most widely used method of hierarchical factor analysis, orthogonalizes the entire factor hierarchy, resulting in factors that are uncorrelated both between and within levels. The Schmid-Leiman procedure provides the loading of each test on the uncorrelated factors at each hierarchical level. The Schmid-Leiman $g$ has been found to be highly related to the $g$ obtained by other factor analysis methods, both in terms of congruence coefficients and the variance accounted for by $g$ in the test battery (Jensen \& Weng, in press; Ree \& Earles, 1991).

\section{METHOD}

Discussion of the subjects, procedures, and experimental tasks, as well as the complete zero-order correlation matrix upon which these analyses are based, can be found in Naglieri et al. (1991). The confirmatory factor analyses were conducted by the generalized-least-squares (GLS) method in the LISREL VI software package (Jöreskog \& Sörbom, 1984). Naglieri et al. (1991), in contrast, used SIMPLIS, a simplified version of LISREL for microcomputers (Jöreskog \& Sörbom, 1987). Both programs are used to examine the structural relationships among variables specified by a model; and both provide a test of the "goodness of fit" of the overall model to the data. The results with LISREL and SIMPLIS will be quite similar.

One of the most important considerations before conducting a confirmatory factor analysis is sample size. As Good and Lane (1990) state, "small sample size affects stability of parameter estimates, the power of hypothesis tests, and the interpretation of goodness-of-fit indices" (pp. 500-501). In order to obtain trustworthy results, according to one rule of thumb, the sample size should be at least five times the number of free parameters to be estimated in the model (Bentler, 1989). Naglieri et al. (1991) report results for two independent samples. The Grades 5-12 group has 10 variables and 132 subjects. The Grades K-2 group, however, has 9 variables and only 73 subjects. A confirmatory factor analysis on 9 variables that allows all factor loadings and residuals to be freely estimated has at least 18 free parameters ( 9 factor loadings and 9 residual variances), not including possible interfactor correlations. Therefore, due to the insufficient sample size of the Grades K-2 group for these analyses, only the data for the Grades 5-12 group were used.

The analyses were conducted in several steps. First, the fit provided by each of the a priori specified models was examined by submitting the correlation matrix to a confirmatory factor analysis, by means of the GLS method of the LISREL VI program. In these analyses the subtests were allowed to load on only one factor and the factors were allowed either to be correlated or to load on a second-order $g$ factor, depending on the model. Next, the following 
indices were examined to evaluate the fit of each model: (1) the chi-squares $\left(\chi^{2}\right)$ goodness-of-fit test; (2) the ratio of $\chi^{2}$ to degrees of freedom $\left(\chi^{2} / d f\right) ;(3)$ the adjusted goodness-of-fit index (AGFI); (4) the root mean square residual (RMR); and (5) the Bentler-Bonnet non-normed fit index (BBNNFI; Bentler \& Bonnet, 1980). The $\chi^{2}$ goodness-of-fit statistic provides a test of the overall fit of the model to the data. Nonsignificant values of $\chi^{2}$ indicate good fit. Also indicative of good fit are $\chi^{2} / d f$ ratios under 2.00. The variability accounted for by the model is estimated by the AGFI, with values near 1 indicating good fit. The RMR measures the discrepancy between the observed correlations and the reproduced correlations based on the model estimates. The BBNNFI is used to estimate the relative improvement of each model over the null independence model, which assumes all variables to be independent, with values near 1 indicating good model fit. The BBNNFI is comparable to the Tucker-Lewis Index (TLI) used by Naglieri et al. (1991). As Naglieri et al. (1991) determined relative improvement over the $g$ model instead of the null independence model, the BBNNFI was calculated both ways. In addition to the evaluation of these goodness-of-fit indices for each model, a statistical test of the $\chi^{2}$ difference between models was conducted to determine the appropriateness of releasing or imposing any restriction on the parameters. Lastly, the first-order matrix of the revised (PA)SS model was submitted to the Schmid-Leiman (1957) orthogonalization procedure to examine the second-order $g$.

\section{RESULTS}

The results of the confirmatory factor analyses are reported in Table 1. The BBNNFI values reported in Table 1 reflect the improvement of each model over the null independence model. The results for the original PASS model are comparable to those obtained by Naglieri et al. (1991). The goodness-offit $\chi^{2}$ statistic was nonsignificant $\left(\chi^{2}(29)=30.15, p=.407\right)$, indicating adequate model fit. The other goodness-of-fit indices also suggest that the

Table 1

Confirmatory Factor Analysis Results

\begin{tabular}{lccccccc}
\hline Model $^{\mathrm{a}}$ & $\chi^{2}$ & $d f$ & $p$ & $\chi^{2} / d f$ & AGFI & RMR & BBNNFI \\
\hline (PA)SS & 32.67 & 32 & .434 & 1.02 & .914 & .063 & .985 \\
PASS & 30.15 & 29 & .407 & 1.04 & .913 & .056 & .970 \\
PASS $+g$ & 35.44 & 31 & .267 & 1.14 & .904 & .063 & .894 \\
Null & 104.44 & 45 & .000 & 2.32 & .805 & .317 & - \\
\hline
\end{tabular}

Note. $N=132$.

${ }^{a}(\mathrm{PA}) \mathrm{SS}=$ Simultaneous, Successive, and Planning/Attention (combined) first-order factors; PASS $=$ Planning, Attention, Simultaneous, and Successive first-order factors; PASS $+g=$ Planning, Attention, Simultaneous, and Successive first-order factors, plus hierarchical $g ;$ Null $=$ null independence model. 
model fits the data reasonably well $\left(\chi^{2} / d f\right.$ ratio $=1.04 ; \mathrm{AGFI}=.913 ; \mathrm{RMR}$ $=.056 ; \mathrm{BBNNFI}=.970$ ). The standardized GLS estimates (with factor variances scaled to 1) for the original PASS model are displayed in Table 2. These results are also shown in Figure 1. All the PASS tests load significantly on their respective factors $(p<.05)$. The interfactor correlations are also significant $(p<.05)$, excepting the correlation between the Attention and Successive factors. The correlation between the Planning and Attention factors is +1.06 , which indicates that the factor solution provided by the PASS model is improper. As Jöreskog and Sörbom (1989) state, "Parameter estimates should have the right sign and size. Examples of unreasonable values of parameter estimates ... [include] . . correlations which are larger than one in magnitude" ( p. 41). Although it is possible that LISREL overestimated the true correlation between the Planning and Attention factors, it seems reasonable to assume, on the basis of this estimate, that the true interfactor correlation is nevertheless quite high.

In the PASS $+g$ hierarchical model, the goodness-of-fit $\chi^{2}$ was also nonsignificant $\left(\chi^{2}(31)=35.44, p=.267\right)$, indicating satisfactory model fit. The other goodness-of-fit indices substantiate that the hierarchical model provides a good fit to the data $\left(\chi^{2} / d f\right.$ ratio $=1.14 ;$ AGFI $=.904 ; \mathrm{RMR}=.063$;

Table 2

Standardized Factor Loadings and Interfactor Correlations for the PASS Model

\begin{tabular}{|c|c|c|c|c|}
\hline \multirow[b]{2}{*}{ Variable } & \multicolumn{4}{|c|}{ Factor } \\
\hline & Simultaneous & Successive & Planning & Attention \\
\hline \multicolumn{5}{|l|}{ PASS Test $^{a}$} \\
\hline MAT & .730 & & & \\
\hline DC & .629 & & & \\
\hline FM & .645 & & & \\
\hline SRQ & & .907 & & \\
\hline SWR & & .562 & & \\
\hline VS & & & .472 & \\
\hline $\mathrm{PC}$ & & & .667 & \\
\hline $\mathrm{MN}$ & & & .607 & \\
\hline SAR & & & & .573 \\
\hline SAE & & & & .314 \\
\hline \multicolumn{5}{|l|}{$\begin{array}{l}\text { Interfactor } \\
\quad \text { correlations }\end{array}$} \\
\hline Simultaneous & 1.00 & & & \\
\hline Successive & .462 & 1.00 & & \\
\hline Planning & .806 & .426 & 1.00 & \\
\hline Attention & .892 & .260 & 1.06 & 1.00 \\
\hline
\end{tabular}




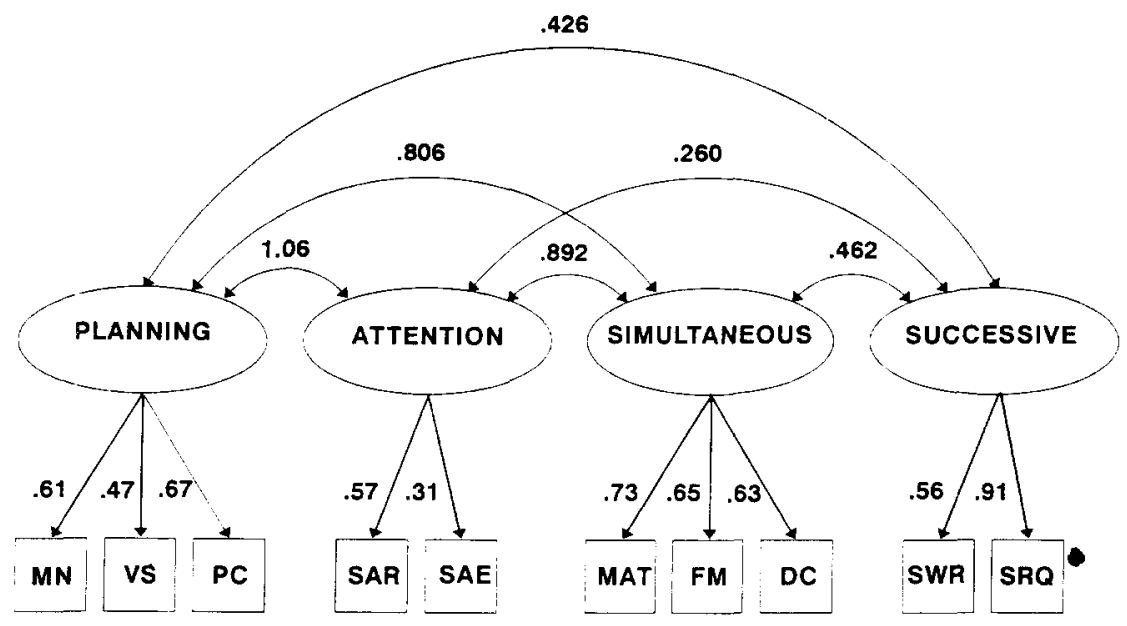

Figure 1. Standardized factor loadings and interfactor correlations for the PASS model. Ellipses are latent constructs; squares are measured variables. Correlations are indicated by two-headed arrows. MAT $=$ Matrices, $\mathrm{DC}=$ Design Construction, $\mathbf{F M}=$ Figure Memory, SRQ = Sentence Repetition and Questions, $\mathbf{S W R}=$ Successive Word Recall, VS = Visual Search, $\mathbf{P C}=$ Planned Connections, $\mathbf{M N}=$ Matching Numbers, SAR = Selective Attention-Receptive, SAE = Selective Attention-Expressive.

BBNNFI $=.894$ ), although perhaps not quite good as that provided by the PASS model. This slight reduction in fit is expected mathematically, however. When more structure is imposed on the data, the resulting fit is always worse. Hence, in comparisons between hierarchical and nonhierarchical models such as these, the $\chi^{2}$ difference is a particularly important index of relative fit. Table 3 presents the results of the statistical tests of the $\chi^{2}$ difference between the three models. The $\chi^{2}$ difference between the PASS and PASS $+g$ models was not significant $\left(\chi^{2}(2)=5.29, p>.05\right)$. According to the principle of

Table 3

Summary of $\chi^{2}$ Differences

\begin{tabular}{lccc}
\hline Model contrast $^{\text {a }}$ & $\chi^{2}$ & $d f$ & $p^{*}$ \\
\hline PASS vs. PASS $+g$ & 5.29 & 2 & NS \\
PASS vs. (PA)SS & 2.52 & 3 & NS \\
$($ PA)SS vs. PASS $+g$ & 2.77 & 1 & NS \\
\hline
\end{tabular}

${ }^{a}$ PASS = Planning, Attention, Simultaneous, and Successive first-order factors; PASS $+g=$ Planning, Attention, Simultaneous, and Successive first-order factors, plus hierarchical $g$; (PA)SS = Simultaneous, Successive, and Planning/Attention (combined) first-order factors.

*All $p>.05$. 
Table 4

Standardized Factor Loadings and Interfactor Correlations for the PASS $+g$ Hierarchical Model

\begin{tabular}{|c|c|c|c|c|}
\hline \multirow[b]{2}{*}{ Variable } & \multicolumn{4}{|c|}{ Factor } \\
\hline & Simultaneous & Successive & Planning & Attention \\
\hline \multicolumn{5}{|l|}{ PASS test ${ }^{a}$} \\
\hline NAT & .719 & & & \\
\hline DC & .629 & & & \\
\hline FM & .657 & & & \\
\hline SRQ & & .927 & & \\
\hline SWR & & .497 & & \\
\hline VS & & & .463 & \\
\hline PC & & & .671 & \\
\hline $\mathrm{MN}$ & & & .593 & \\
\hline SAR & & & & .543 \\
\hline SAE & & & & 309 \\
\hline \multicolumn{5}{|c|}{ Interfactor Correlations/Factor Loadings on $g^{*}$} \\
\hline Simultaneous & $1.00 / .877^{*}$ & & & \\
\hline Successive & .381 & $1.00 / .434^{*}$ & & \\
\hline Planning & .848 & .420 & $1.00 / .967^{*}$ & \\
\hline Attention & .971 & .480 & 1.07 & $1.00 / 1.107^{*}$ \\
\hline
\end{tabular}

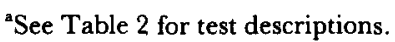

Occam's razor, when the $\chi^{2}$ difference between two models is nonsignificant, the most parsimonious model is superior. In LISREL, parsimony is reflected in the degrees of freedom $(d f)$, with greater parsimony corresponding to more $d f$. The $d f$ of the PASS and PASS $+g$ models are 29 and 31, respectively, indicating that the PASS $+g$ model is more parsimonious than the PASS model and therefore better. Table 4 presents the standardized GLS estimates for the PASS $+g$ hierarchical model. All the factor loadings are significant $(p<.05)$. In addition, each of the four first-order factors loads significantly on the hierarchical $g(p<.05)$, indicating that they share considerable variance. The variance contained in the first-order factors is what remains after the variance accounted for by $g$ has been partialed out. Finally, the correlation between the Planning and Attention factors exceeds 1.00 , which indicates that the PASS $+g$ hierarchical model also provides an improper factor solution for these data.

The goodness-of-fit indices for the (PA)SS model are generally better than those for both the original PASS model and the PASS $+g$ hierarchical model (goodness-of-fit $\chi^{2}(32)=32.67, p=.434 ; \chi^{2} / d f$ ratio $=1.02 ;$ AGFI $=$ .914 ; RMR $=.063$; BBNNFI $=.985)$. These results suggest that the revised (PA)SS model provides the best fit to the data. Notwithstanding the apparent superior fit of the (PA)SS model, the $\chi^{2}$ difference between it and the original PASS model was not significant $\left(\chi^{2}(3)=2.52, p>.05\right)$; nor was the $\chi^{2}$ difference between the (PA)SS model and the PASS $+g$ hierar- 
chical model $\left(\chi^{2}(1)=2.77, p>.05\right)$. The (PA)SS model has the most $d f$, however, indicating that it is the most parsimonious and therefore the best of the three models in describing these data. Table 5 presents the standardized GLS estimates for the (PA)SS model. The PASS tests load significantly on their respective factors $(p<.05)$. In addition, all interfactor correlations are significant $(p<.05)$, indicating the presence of a substantial $g$ among the revised (PA)SS factors.

A final confirmatory factor analysis was conducted on a one-factor model, referred to by Naglieri et al. (1991) as the $g$ model. Each test was allowed to load on this factor. Not surprisingly, the one-factor model was found to provide a poor fit to the data (goodness-of-fit $\chi^{2}(35)=58.06, p=.008 ; \chi^{2} / d f$ ratio $=1.66 ;$ AGFI $=.861 ; \mathrm{RMR}=.107 ; \mathrm{BBNNFI}=.500)$. For comparison with the results of Naglieri et al. (1991), the BBNNFI for each of the three models was calculated with the $g$ model. These BBNNFI values were .788 for the PASS $+g$ model, .939 for the PASS model, and .970 for the revised (PA)SS model. These results further indicate that the revised (PA)SS model provides the most improved fit over the one-factor model.

In addition to the LISREL analyses, the first-order matrix of the (PA)SS model was submitted to the Schmid-Leiman (1957) orthogonalization procedure to examine the second-order $g$ factor, using the squared multiple correlations as communality estimates. The results of this analysis are presented in

Table 5

Standardized Factor Loadings and Interfactor Correlations for the Revised (PA)SS Model

\begin{tabular}{llll}
\hline & \multicolumn{3}{c}{ Factor } \\
\cline { 2 - 4 } Variable & Simultaneous & Successive & $\begin{array}{r}\text { Planning/ } \\
\text { Attention }\end{array}$ \\
\hline PASS test & a & & \\
MAT & & & \\
DC & .717 & & \\
FM & .643 & .947 & \\
SRQ & .654 & .494 & .464 \\
SWR & & & .669 \\
VS & & & .601 \\
PC & & & .618 \\
MN & & & .318 \\
SAR & & & \\
SAE & & & \\
Interfactor correla- & & & \\
tions & & & \\
Simultaneous & 1.00 & & \\
Successive & .454 & & \\
Planning/ & & & \\
$\quad$ Attention & .813 & & \\
\hline
\end{tabular}

asee Table 2 for test descriptions. 
Table 6. They are also displayed in Figure 2. The second-order $g$ accounts for $59.3 \%$ of the common factor variance among the PASS tests, which is more than that accounted for by the Simultaneous (7.4\%), Successive $(22.4 \%)$ and Planning/Attention (10.9\%) factors combined. The loading of each firstorder factor on hierarchical $g$ is .884 for the Simultaneous factor, .464 for the Successive factor, and .843 for the Planning/Attention factor. The substantial $g$ among the PASS tests is further revealed by the fact that each test's loading on $g$ is higher than its residualized first-order factor loading, excepting the two tests that loaded on the Successive factor (viz., Successive Word Recall and Sentence Repetition and Questions). The communality $\left(h^{2}\right)$ of each test is also reported in Table 6 . These values are generally rather small, compared with conventional intelligence tests (cf. Blaha \& Wallbrown, 1982), especially for some of the planning and attention tasks, indicating that considerable variance among these tests is related to specificity and error.

\section{DISCUSSION}

This study used confirmatory factor analysis to compare the relative fit to the data provided by the PASS model with that of two alternative models of mental ability that were not examined by Naglieri et al. (1991). The results indicate that the original PASS model does not provide a significantly better description of the correlational structure of the tests used to operationalize the PASS theory. In fact, the PASS model appears to provide an improper factor

Table 6

Results of the Schmid-Leiman Hierarchical Factor Analysis of the Revised (PA)SS Model

\begin{tabular}{|c|c|c|c|c|c|}
\hline \multirow[b]{2}{*}{ Variable } & \multirow[b]{2}{*}{$g$} & \multicolumn{4}{|c|}{ Residualized factor loadings } \\
\hline & & Simultaneous & Successive & $\begin{array}{l}\text { Planning/ } \\
\text { Attention }\end{array}$ & $h^{2}$ \\
\hline \multicolumn{6}{|l|}{ PASS test } \\
\hline MAT & .634 & .335 & & & .514 \\
\hline DC & .568 & .301 & & & .413 \\
\hline FM & .578 & .306 & & & .428 \\
\hline SRQ & .439 & & .839 & & .897 \\
\hline SWR & .229 & & .438 & & .244 \\
\hline VS & .391 & & & .250 & .215 \\
\hline $\mathrm{PC}$ & .564 & & & .360 & .448 \\
\hline $\mathrm{MN}$ & .507 & & & .323 & .361 \\
\hline SAR & .521 & & & .332 & .382 \\
\hline SAE & .268 & & & .171 & .101 \\
\hline CFV & 2.375 & 0.296 & 0.896 & 0.436 & \\
\hline$\%$ Variance & $59.3 \%$ & $7.4 \%$ & $22.4 \%$ & $10.9 \%$ & \\
\hline \multicolumn{6}{|c|}{ Factor loading on } \\
\hline
\end{tabular}

Note. See Table 2 for test descriptions. $h^{2}=$ Communality. CFV = Common factor variance. 


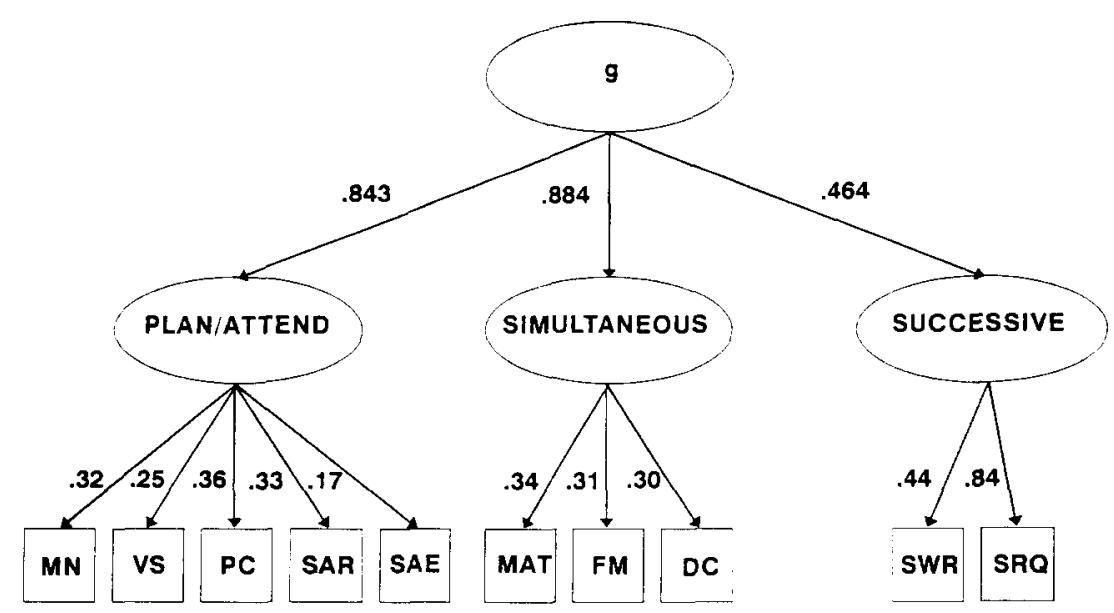

Figure 2. Standardized factor loadings and interfactor correlations for the (PA)SS $+g$ hierarchical model. Ellipses are latent constructs; squares are measured variables. See Figure A-1 for test descriptions. Plan/Attend = combined Planning and Attention factor.

solution for these data. According to the various goodness-of-fit indices examined in this study, the best fit to the data was provided by the (PA)SS model, which has separate Simultaneous and Successive factors and a combined Planning/Attention factor. The (PA)SS model also has the added theoretical advantage that it is more parsimonious than the original PASS model.

A substantial psychometric $g$ was also extracted from the battery of PASS tests, as suspected by Lambert (1990) and by Reschly and Wilson (1990). Indeed, the results of this study indicate that the PASS battery of tests primarily measures $g$. The second-order $g$ of the (PA)SS model accounted for $59.3 \%$ of the common factor variance among the PASS tests; and the loading of each test on $g$ was higher than its residualized first-order factor loading, with the exception of the two successive processing tests. Moreover, the $g$ of the PASS tests accounted for considerably more variance than the $g$ of conventional IQ tests. For example, the total variance accounted for by psychometric $g$ was $44 \%$ on the K-ABC (Jensen, 1984) and $43 \%$ on the WISG-III (Sattler, 1992).

In addition to these results, examination of the $g$-loadings of the PASS tests suggests that the $g$ factors extracted from the PASS and conventional IQ tests are similar. ${ }^{1}$ For example, among the PASS tests, Matrices has the highest

\footnotetext{
'The similarity of the psychometric $g$ extracted from different test batteries can be determined empirically by calculating the coefficient of congruence between the $g$-loadings of the tests that are included across batteries (e.g., Jensen \& Weng, 1994; Naglieri \& Jensen, 1987). Unfortunately, due to the contemporaneousness of many of the PASS tests, and the fact that this is the first study to examine the PASS $g$, the data are not available for the calculation of this statistic. The interpretation of the comparability of the PASS $g$ in the present study should therefore be regarded as a tentative hypothesis until substantiated.
} 
loading on $g$. This test was developed from the Matrix Analogies Test (MAT) (Naglieri, 1985), which, according to Nicholson (1990), correlates +.71 with Raven's Progressive Matrices (Raven, 1956), long considered one of the best "marker" tests of $g$ ( see Jensen, 1980). Conversely, Word Recall has the lowest loading on $g$ of all the PASS tests. Tests of short-term sequential memory such as this have been found to be minimally related to $g$ (e.g., Jensen, 1984; Marshalek, Lohman, \& Snow, 1983; Naglieri \& Jensen, 1987). Given that the pattern of the PASS tests' $g$-loadings generally conforms with the $g$-loadings of similar tests in other batteries, the results of this study therefore suggest that the $g$ of the PASS tests is comparable to the $g$ of conventional IQ tests.

Contrary to the contention of Naglieri et al. (1990) that "planning and attention have emerged as separate factors distinct from simultaneous and successive coding" (p. 431), the results of this study also suggest that planning and attention are not "functionally distinct," as claimed, but functionally indistinguishable, at least with children in Grades 5-12 and with this particular battery of tasks. These results do not contradict Telzrow's (1990) assertion that "the inclusion of [attention] as a separate process in a theoretical model of intelligence does not appear consistent with the literature" (p. 348). Although one could argue that the "structural architecture" underlying the Planning and Attention factors is distinct, as indicated by research in neurology and experimental psychology, the correlational evidence presented here suggests that individual differences in planning and attention are related to the same underlying source of variance. When conceptually distinct processes such as these are highly correlated, the presence of some more fundamental level of processes, presumably neurological, that is shared by these processes is implicated (Jensen, 1987c). Contemporary research on the nature of such biological underpinnings of individual differences in intelligence has made remarkable progress in recent years (e.g., see the special issue of Intelligence, 1992, Volumes 3-4). Of course, another plausible interpretation of these results is that the planning and attention processes of the PASS model are distinct, but that the tests used to operationalize the PASS constructs fail to adequately distinguish between them. In either case, inclusion of the present battery of planning and attention tests in Das and Naglieri's new measure of mental ability (the Cognitive Assessment System) is suspect. However, as the results of any factor analysis technique depend upon the specific variables that are entered ( see Carroll, 1993), substantial revision of the present battery of tests may lead to different results than those obtained in the present study. Cross-validation of these results on a different data set is also required before firm conclusions regarding the latent structure of the PASS tests can be made.

What does the combined Planning/Attention factor appear to measure? Given that all the planning and attention tasks involve timing, with faster performance resulting in higher scores, this factor seems to reflect individual differences in response speed. It is important to distinguish between response speed and speed of information processing. Numerous recent studies have demon- 
strated that speed of processing is integrally related to individual differences in IQ scores (e.g., Carroll, 1991; Kranzler \& Jensen, 1989, 1991; Larson \& Saccuzzo, 1989), which, according to Naglieri et al. (1990), are largely a function of the simultaneous and successive processes. In addition, speed of processing has been found to be related to scores on both timed and untimed IQ tests (Vernon \& Kantor, 1986; Vernon, Nador, \& Kantor, 1985). Therefore, all three revised (PA)SS factors likely reflect to some degree the speed of information processing. The Planning/Attention factor, however, can be distinguished from the Simultaneous and Successive factors on the basis of dissimilarities in response requirements, the primary difference being an emphasis on responding quickly in the planning/attention tasks.

Lastly, it is important to distinguish between exploratory and confirmatory factor analysis. Exploratory factor analysis is used to investigate the correlational structure of empirical data. It is most useful in the early stages of research, when initial hypotheses about the latent sources of variance and covariance in the data are generated. Although exploratory factor analysis does not provide statistical tests of the number of factors or their factor loadings, the hypotheses that are developed by its results can be tested by collecting additional data and subjecting these results to multivariate methods, such as LISREL. In confirmatory factor analysis, in contrast, models of the data's latent structure are constructed according to specific theories or hypotheses and tested to determine how well they describe or fit the data. Jöreskog and Sörbom (1989) state that "the LISREL methodology works best when it is applied to designed studies based on a definite theory and with a clear objective" (p. 224). They further state that LISREL is not very useful "in exploratory situations with many variables and weak or non-existing substantive theory" (p. 225). Thus, both exploratory and confirmatory factor analysis perform necessary functions in the development of theories of mental ability. It is important to note that the aim of this study was not to develop a theory of the structure of mental ability, but to further investigate the factor structure of a battery of tasks hypothesized to measure the PASS constructs. As these results do not support the superiority of the PASS model over other conceptualizations of intelligence in describing these data, further refinement of the PASS theory or tests, or perhaps both, appears to be necessary.

\section{ACKNOWLEDGMENTS}

We thank Dr. Arthur Jensen and three anonymous reviewers for their valuable comments on this manuscript.

\section{REFERENCES}

Bentler, P. M. (1989). EQS structural equations program manual. Los Angeles: BMDP Statistical Software. 
Bentler, P. M., \& Bonnet, D. G. (1980). Significance tests and goodness of fit in the analysis of covariance structures. Psychological Bulletin, 85, 588-606.

Blaha, J., \& Wallbrown, F. H. (1982). Hierarchical factor structure of the Wechsler Adult Intelligence Scale-Revised. Journal of Consulting and Clinical Psychology, 50, 652-660.

Carroll, J. B. (1991). No demonstration that $g$ is not unitary, but there's more to the story: Comment on Kranzler and Jensen. Intelligence, 15, 423-436.

Carroll, J. B. (1993). Human cognitive abilities: $A$ survey of factor-analytic studies. New York: Cambridge University Press.

Das, J. P. (1992). Beyond a unidimensional scale of merit. Intelligence, 16, 137-150.

Das, J. P., \& Varnhagen, C. K. (1986). Neuropsychological functioning and cognitive processing. Child Neuropsychology, 1, 117-140.

Good, R. H., \& Lane, S. (1990). Confirmatory factor analysis of the K-ABC and WISC-R for at-risk students: A comparison of hierarchical models. School Psychology Review, 19, 492-504.

Gustafsson, J. (1984). A unifying model for the structure of intellectual abilities. Intelligence, 8, 179-203.

Jensen, A. R. (1974). Interaction of level I and level II abilities with race and socioeconomic status. Journal of Educational Psychology, 66, 99-111.

Jensen, A. R. (1980). Bias in mental testing. New York: Free Press.

Jensen, A. R. (1984). The black-white difference on the K-ABC: Implications for future tests. Journal of Special Education, 18, 377-408.

Jensen, A. R. (1986). g: Artifact or reality? Journal of Vocational Behavior, 7, 301-331.

Jensen, A. R. (1987a). Psychometric $g$ as a focus of concerted research effort. Intelligence, 11, 193-198.

Jensen, A. R. (1987b). Differential psychology: Towards consensus. In S. Modgil \& C. Modgil (Eds. ), Arthur Jensen: Consensus and controversy. New York: Falmer Press.

Jensen, A. R. (1987c). Process differences and individual differences in some cognitive tasks. Intelligence, 11, 107-136.

Jensen, A. R., \& Weng, L. (1994). What is a good g? Intelligence, 18, 231-258.

Jöreskog, K. G., \& Sörbom, D. (1984). LISREL VI: Analysis of linear structural relationships by the method of maximum likelihood: Users guide. Mooresville, IN: Scientific Software.

Jöreskog, K. G., \& Sörbom, D. (1987). SIMPLIS: Estimating linear structural relationships the easy way using two-stage least squares. Chicago: International Educational Services.

Jöreskog, K. G., \& Sörbom, D. (1989). LISREL VII: A guide to the program and applications (2nd ed.). Chicago: SPSS Inc.

Kaufman, A. S., \& Kaufman, N. L. (1983). Kaufman Assessment Battery for Children. Circle Pines, MN: American Guidance Service.

Kranzler, J. H., \& Jensen, A. R. (1989). Inspection time and intelligence: A metaanalysis. Intelligence, 13, 329-347.

Kranzler, J. H., \& Jensen, A. R. (1991). The nature of psychometric g: Unitary process or a number of independent processes? Intelligence, 15, 397-422.

Lambert, N. M. (1990). Consideration of the Das-Naglieri cognitive assessment system. Journal of Psychoeducational Assessment, 8, 338-343.

Larson, G. E., \& Saccuzzo, D. P. (1989). Cognitive correlates of general intelligence: Toward a process theory of $g$. Intelligence, 13, 5-32.

Luria, A. R. (1966). Human brain and psychological processes. New York: Harper \& Row.

Luria, A. R. (1973). The working brain: An introduction to neuropsychology. New York: Basic Books.

Luria, A. R. (1980). Higher cortical functions in man (2nd ed.). New York: Basic Books.

Marshalek, B., Lohman, D. F., \& Snow, R. E. (1983). The complexity continuum in the radex and hierarchical models of intelligence. Intelligence, 7, 107-127. 
Naglieri, J. A. (1985). Matrix Analogies Test. San Antonio, TX: Psychological Corporation.

Naglieri, J. A. (1989). A cognitive processing theory for the measurement of intelligence. Educational Psychologist, 24, 185-206.

Naglieri, J. A., \& Das, J. P. (1988). Planning-arousal-simultaneous-successive (PASS) cognitive processes: A model for intelligence. Journal of School Psychology, $27,347-364$.

Naglieri, J. A., \& Das, J. P. (1990). Planning, attention, simultaneous, and successive (PASS): A model for assessment. Journal of Psychoeducational Assessment, 36, 3548 .

Naglieri, J. A., Das, J. P., \& Jarman, R. F. (1990). Planning, attention, simultaneous, successive (PASS) cognitive processes as a model for assessment. School Psychology Review, 19, 423-442.

Naglieri, J. A., Das, J. P., Stevens, J. J., \& Ledbetter, M. F. (1991). Confirmatory factor analysis of planning, attention, simultaneous, and successive cognitive processing tasks. Journal of School Psychology, 29, 1-17.

Naglieri, J. A., \& Jensen, A. R. (1987). Comparison of black-white differences on the WISC-R and K-ABC: Spearman's hypothesis. Intelligence, 11, 21-43.

Naglieri, J. A., Prewett, P., \& Bardos, A. N. (1989). An exploratory study of planning, attention, simultaneous, and successive cognitive processes. Journal of School Psychology, 27, 423-442.

Nicholson, C. I. (1990). Matrix Analogies Test. Diagnostique, 15, 115-123.

Raven, J. C. (1956). Coloured Progressive Matrices: Sets $A, A b, B$. London: H. K. Lewis.

Ree, M. J., \& Earles, J. A. (1991). The stability of $g$ across different methods of estimation. Intelligence, 15, 271-278.

Reschly, D. J., \& Wilson, M. S. (1990). Cognitive processing versus traditional intelligence: Diagnostic utility, intervention implications, and treatment validity. Journal of School Psychology, 19, 443-458.

Sattler, J. M. (1992). Assessment of children (rev. 3rd ed.). J. M. Sattler, Publisher: San Diego.

Schmid, J., \& Leiman, J. M. (1957). The development of hierarchical factor solutions. Psychometrika, 22, 53-61.

Spearman, C. (1927). The abilities of man. London: MacMillan.

Telzrow, C. F. (1990). Does PASS pass the test? A critique of the Das-Naglieri cognitive assessment system. Joumal of Psychoeducational Assessment, 8, 344-355.

Unheim, J. O., \& Gustaffson, J. (1987). The hierarchical organization of cognitive abilities. Restoring general intelligence through the use of linear structural relations (LISREL). Multivariate Behavioral Research, 22, 149-171.

Vernon, P. A. (1981). Level I and level II: A review. Educational Psychologist, 16, 4564.

Vernon, P. A., \& Kantor, L. (1986). Reaction time correlations with intelligence test scores obtained under either timed or untimed conditions. Intelligence, 10, 315-330.

Vernon, P. A., Nador, S., \& Kantor, L. (1985). Reaction times and speed-ofprocessing: Their relationship to timed and untimed measures of intelligence. Intelligence, 9, 357-374.

Wechsler, D. (1991). Wechsler Intelligence Scales for Children-Third Edition. New York: Psychological Corporation. 